\title{
Zum 75. Geburtstag von Dr. Reinhard Gaedike
}

\author{
EckHard K. Groll, Christian Kutzscher \& Andreas Taeger
}

Senckenberg Deutsches Entomologisches Institut, Eberswalder Straße 90, 15374 Müncheberg

Published on 2017-12-08

DOI: $10.21248 /$ contrib.entomol.67.2.193-194;

Heute ist die Digitalisierung in aller Munde. Aber wann begann das alles? Woher kommen die Daten? Im DEI begann diese Entwicklung bereits Anfang der 1990er-Jahre.

Unmittelbar nach meinem Eintritt ins DEI suchten einige Wissenschaftler Kooperationspartner in anderen Museen, diskutierten Ideen zum Einsatz von Computern im Institut und formulierten Projekte zur Finanzierung. Reinhard, als Leiter der Bibliothek und Autor bedeutender gedruckter Datensammlungen, war von der Zukunft der digitalen Verarbeitung entomologischer Daten überzeugt. Dank der großzügigen Ausstattung mit Computertechnik durch das Bundesministerium des Innern konnten wir auch sofort anfangen. Bereits im Jahresbericht der Projektgruppe Entomologie (ehem. Deutsches Entomologisches Institut) von 1992 vermeldeten wir den „Aufbau von Computer-Datenbanken und [die] Übernahme der Daten aus den traditionellen Datensammlungen des ehemaligen DEI“.

In den folgenden Jahren entstanden der „Index Novus Litteraturae Entomologicae“ (http://sdei.senckenberg. de/index), die umfangreichste und genaueste Dokumentation der entomologischen Literatur vom Anfang bis einschließlich 1864, die „Biografien der Entomologen der Welt" (http://sdei.senckenberg.de/biographies) und mit der Digitalisierung der Bestandskartei unserer Spezialbibliothek B15 auch der Vorläufer unseres modernen OPAC. Dabei waren Reinhards Erfahrungen, z. B. durch die Ergänzungen und Berichtigungen zu Walter Horns und Sigmund Schenklings Index Litteraturae oder durch die Publikation der Bibliographie der Bestimmungstabellen europäischer Insekten, von unschätzbarem Wert. Unermüdlich hat er bibliografische Angaben recherchiert und in Datenbanken eingegeben. Selbst im Ruhestand, in dem er sich endlich intensiver um die Kleinschmet- terlinge kümmern kann, hat er die Daten des „Index“ korrigiert.

In der vorliegenden Zeitschrift „Contributions to Entomology = Beiträge zur Entomologie“ erscheinen die Artikel seit diesem Jahr mit je einer ergänzenden Liste der behandelten Taxa. Dank Reinhards fleißiger Unterstützung - er hat über 7000 taxonomische Handlungen erfasst - konnten auch alle archivierten Artikel mit dieser Liste versehen werden.

Die fruchtbare Zusammenarbeit mit Reinhard über viele Jahre ermöglichte mir ein spannendes Arbeitsleben im „Informationszentrum Entomologie“. Gemeinsam haben wir in der Herbstschen „entomologischen Republik“ gewirkt (Herbst, J. F. W. 1781: Plan zu einer entomologischen Republik. - Neues Magazin für die Liebhaber der Entomologie (Fuessly, J. C. [Hrsg.]), Zürich und Winterthur - 1 (1) 1-12). Dafür bedanke ich mich anlässlich seines 75. Geburtstages und wünsche Gesundheit.

Eckhard

Reinhard Gaedike war von Anbeginn meiner Arbeit als Zoologischer Präparator am Deutschen Entomologischen Institut bis zu seiner Berentung über zwanzig Jahre mein unmittelbarer Vorgesetzter und wichtigster Ansprechpartner. Das hat geprägt und gern erinnere ich mich daran zurück. Unaufdringlich, aber doch bestimmt, nahm er sich meiner an und half mir im Institut „laufen zu lernen“. Die Neuaufstellung, einschließlich der Eingliederung neu erworbener Privatsammlungen in die Kleinschmetterlingssammlung, gehörte zu meinen Hauptaufgaben. 
Wann immer es sich einrichten ließ, nahm er mich auf Exkursionen und zu Fachtagungen mit, wobei ich nicht nur seine Forschungsobjekte, sondern auch den entomologischen Kollegenkreis näher kennen lernen konnte. Überhaupt stand er der Weiterbildung seiner technischen Mitarbeiter und Mitarbeiterinnen stets aufgeschlossen gegenüber, ermöglichte uns beispielsweise die Teilnahme an Präparationstagungen und setzte sich nicht zuletzt auch für meine Zulassung zum Fachschulfernstudium in der Fachrichtung „Präparator für naturwissenschaftliches Sammlungsgut“ ein.

Nachhaltig dankbar bin ich für die großzügige Gewährung von Freiräumen, in denen ich meinem eigenen entomologischen Steckenpferd, den Flöhen (Siphonaptera), nachgehen konnte. Dabei wurde diese Passion nicht nur geduldet, sondern auch aktiv gefördert, z. B. durch die gezielte Vermittlung von Kontakten. Bereits für die Abschlussarbeit meiner Lehre zum Zoologischen Präparator wurde mir ein flohkundliches Thema angeboten, das von Reinhard mit erdacht worden war.

Als sehr ehrenhaft und vorbildlich habe ich immer auch Reinhards Achtung vor den Altvorderen unseres Instituts empfunden, insbesondere die vor Walther Horn. Die Würdigungen von dessen Verdiensten um das Institut, die mich seit meiner ersten Hausführung durch Reinhard begleitet haben, weckten in mir das Interesse nicht nur an der Person Horns, sondern auch an der Institutsgeschichte allgemein und bewirkten ein tiefes Identifikationsgefühl mit dieser „meiner“ Forschungsstätte.

Dass Reinhard mittlerweile seit zehn Jahren im Ruhestand ist, habe ich vollkommen verdrängt. Wir sehen uns zwar seither nicht mehr so oft, dennoch erhalte ich nach wie vor Arbeitsaufträge von ihm, per Post, per Telefon oder per E-Mail. Es ist fast so, als führe er das immer noch unbesetzte Schmetterlingskustodiat weiter. Zusammenfassend kann ich sagen, Reinhard war und ist mein Chef bis heute. Dafür möchte ich an dieser Stelle Danke sagen und ich hoffe und wünsche uns, dass dies noch lang so bleibt!

Christian

Es ist ganz sicher nicht möglich, mit wenigen Worten der Bedeutung Reinhard Gaedikes für das SDEI gerecht zu werden. Die folgenden Zeilen sollen aber wenigstens ein paar Facetten seiner Persönlichkeit beleuchten. Ich lernte Herrn Dr. Gaedike vor über 35 Jahren im damaligen Institut für Pflanzenschutzforschung (Abteilung Taxonomie der Insekten) in Eberswalde-Finow kennen. Reinhard - so hieß er für mich als jungen Hymenopterologen schon bald - war er ein älterer (40 Jahre alter!) Spezialist für einige Gruppen der Kleinschmetterlinge. Es gab also fachlich nur begrenzte Schnittmengen, wenn man mal von allgemeinen taxonomischen oder nomenklatorischen Fragen absieht. Es dauerte aber trotzdem nicht allzu lange, und wir trafen uns regelmäßig zum Skat spielen. Wohlgemerkt nach dem Dienst. Im Rückblick auf die letzten 35 Jahre wird klar, dass es eine Zeit ständiger Veränderungen war. Das geht von grundlegenden Strukturveränderungen des Institutes (Loslösung der Spezialbibliothek B15 von der „Abteilung Taxonomie”), über die politischen Umwälzungen in der DDR Ende der 1980er Jahre und die Wiedervereinigung, ein Jahrzehnt hochgradiger Gefährdung der Existenz des DEI als Forschungsinstitut, die Einbindung des DEI in das ZALF und dem damit einhergehenden Umzug des kompletten Institutes von Eberswalde nach Müncheberg, bis hin zur aktuellen Integration in die SGN als Senckenberg Deutsches Entomologisches Institut.

Was sich in dieser Zeit nicht verändert hat, ist die Verlässlichkeit Reinhards. Während seiner Dienstjahre stellte er sich stets den neuesten Anforderungen, auch wenn diese durchaus nicht immer seinen fachlichen Interessen entsprachen. Als notwendig oder unvermeidbar erkannte Aufgaben erledigte er schnell, effizient und meist „lautlos”. Ich glaube, dass seine Tätigkeit und Präsenz im Hause ein ganz wesentlicher Faktor für das Überleben des DEI in schwierigen Zeiten war. Einer seiner Wahlsprüche - „Man muss immer wissen, wo die größeren Kanonen stehen!” - ist im SDEI zum geflügelten Wort und für viele von uns durchaus Richtschnur des Handelns geworden.

Die Verbundenheit Reinhard Gaedikes mit seinem alten Institut ist ungebrochen, er findet immer wieder die Möglichkeit, von Bonn nach Müncheberg zu kommen. Meist hat er hier vor, fachliche Dinge zu klären. Auch gibt es in Müncheberg immer wieder Fragen, die man gern mit Reinhard besprechen möchte, und deshalb einfach mal schnell zum Telefon greift. Solche Überfälle nimmt er gelassen. Als Rentner war Reinhard viele Jahre Vorsitzender des Fördervereins des DEI. Auch in dieser Funktion konnten wir uns stets auf seine Erfahrung verlassen. Es gibt viele weitere Seiten von Reinhard Gaedike die hier nicht aufscheinen, so z. B. der Familienmensch, fehlende i-Punkte oder die Formel 1. Aber darüber kann ja vielleicht in 25 (gesunden) Jahren von seinen Urenkeln berichtet werden.

Andreas

Für weitere Wortmeldungen hat der Druckraum in diesem Heft nicht mehr gereicht. Deshalb melden sich alle Mitarbeiterinnen und Mitarbeiter des SDEI in diesem kleinen Absatz zu Wort: „Herzlichen Glückwunsch zum Geburtstag, Gesundheit, Schaffenskraft und ein baldiges Wiedersehen im Institut!“. 